\title{
Harnessing the biology of the oviduct for the benefit of artificial insemination
}

W.V. Holt' ${ }^{1}$, R.M.A. Elliott' ${ }^{1}$ A. Fazeli ${ }^{2}$, E. Sostaric ${ }^{2}$, A.S. Georgiou ${ }^{2}$, N. Satake ${ }^{1,3}$, N. Prathalingam ${ }^{3}$ and P.F. Watson ${ }^{3}$

'Institute of Zoology, Regent's Park, London NW1 4RY, UK; ${ }^{2}$ Academic Unit of Reproductive and Developmental Medicine, The University of Sheffield, Level 4, The lessop Wing, Tree Root Walk, Sheffield S1O 2SF, UK; ${ }^{3}$ BBS, Royal Veterinary College, Royal College Street, London NW1 OTU, UK

Spermatozoa fulfil a single role, namely achieving syngamy by transporting the haploid genome to their counterpart gamete, the oocyte. Simple as this may seem, it is fraught with many difficulties, especially in the face of biological processes that enable females to select spermatozoa after they have mated multiply with several males. Conversely, the female reproductive tract sequesters a privileged sperm subpopulation in the oviductal isthmus for variable periods of time, releasing them when the time is opportune for fertilisation. Recent studies of sperm transport in the female reproductive tract suggest that these phenomena involve signalling dialogues between spermatozoa and the female reproductive tract environment. Opportunities for mutual signalling are immense but have received relatively little attention. The oviduct is an organ of crucial significance in modulating sperm function and may be one of the most important sites for determining many aspects of sperm selection and competition. The oviductal environment possesses the potential for enhancing sperm survival, suppressing and activating sperm motility as required, and responds to the arrival of spermatozoa by producing novel proteins. While the biological nature of the sperm-oviduct dialogue is interesting for its own sake, the mechanisms that govern these processes offer opportunities for the improvement of artificial insemination procedures. If oviductal proteins enhance sperm survival, they offer opportunities for the development of long-life semen diluents. Conversely, if we understood the basis of sperm selection we may be able to concentrate on identifying and using only the best sperm subpopulations for improved animal breeding efficiency.

\section{Introduction}

Artificial insemination (Al) is now a fundamental technology for breeding domestic animals, in human infertility treatments and in wildlife conservation programmes for breeding threatened species. The agricultural application of $\mathrm{Al}$ is mainly focused upon the management and im- 
provement of breed genetics, where traits that are identified as desirable can be efficiently distributed through the use of diluted and/or frozen semen. However, current methods for short and long term semen preservation severely compromise the sperm's survival, both in the extenders and in the female reproductive tract, and hence limit the successful application of the technique.

Protection and maintenance of spermatozoa is a natural function of the mammalian oviduct and it would seem obvious that elucidating the mechanisms involved might help identify components or functions of the oviduct that could usefully be harnessed for semen preservation. Of course, the oviduct is also tasked with preparing spermatozoa for fertilisation, a destabilising process which might be expected to enhance sperm death through premature capacitation and acrosome reactions. Under natural conditions this degenerative outcome tends to be suppressed until an appropriate time for fertilisation, implying that the oviduct exerts subtle control over sperm function and achieves a balance between storage and stability on the one hand and activation on the other. We are still ignorant of the way in which this is achieved, even though the topic has been studied for many years. This has partly been due to the difficulty of developing appropriate laboratory models and in vitro approaches; it is inevitable that such model systems are relatively poor substitutes for the real in vivo oviduct, and it has generally been the case that logically directed studies of a living system have been unable to monitor multiple cellular events and processes simultaneously. Modern high throughput techniques in cell biology, especially genomics and proteomics, are now changing the way such studies can be undertaken and therefore we expect to see a transformation of this field in the very near future.

Given the developing status of this field, the aims of this review will be to examine our current knowledge of the way in which spermatozoa and oviduct mutually interact and to provide a critical appraisal of the investigative techniques typically employed in these investigations.

\section{Biological background}

Sperm storage in the female reproductive tract has been extensively documented in a range of vertebrates that includes sharks (Pratt and Tanaka, 1994), salamanders (Sever and Brizzi, 1998) snakes and turtles (Gist and Jones, 1987; Galbraith 1993) as well as many birds and mammals. Some of these species store spermatozoa for several months or even years. The evolutionary development of this remarkable ability provides an interesting parallel to the aims and intentions of biotechnologists who wish to extend the lifespan of spermatozoa, either in a bottle before insemination or within the female reproductive tract after insemination. In both instances the motivation is to uncouple the synchrony between insemination and ovulation. If afforded this capability, animal breeders would be able to inseminate females without worrying too much about accuracy of ovulation prediction, or at the very least would be able to relax the synchrony by several hours without compromising fertility.

In birds, spermatozoa are stored in sperm storage tubules (Bakst, 1993; Birkhead, 1998; Bakst and Vinyard, 2002) and can remain there for several weeks (6-42 days). Prior to fertilisation the spermatozoa relocate to the infundibular region of the oviduct in readiness to meet newly ovulated eggs. Some reptiles store spermatozoa for very long periods indeed; estimates range from several months to several years (Galbraith, 1993). Although these long storage periods allow the females to retain spermatozoa from one season to another, it is also of relevance that some species have succeeded in dissociating the need for synchrony between ovulation and mating. Sperm storage occurs in several different regions within the female reproductive tract, 
ranging from sacs in the infundibular region of the oviduct to crypts in the mucosa of the uterovaginal junction (Gist and Congdon, 1998). Some of these sperm storage regions are ducts of oviductal glands or crypt-like extensions of the oviductal epithelium at the base of mucosal folds (Gist and Jones, 1987; Almeida-Santos et al., 2004). Use of modern genetic markers has confirmed that stored spermatozoa are indeed used for fertilisation, even though other matings may have occurred during the intervening period (Pearse et al., 2001).

Although sperm storage in these species has been described extensively, and its adaptive significance interpreted, few studies have addressed the physiological mechanisms that may be involved. Breque et al. (2003) observed that avian semen contains a cocktail of substances, including high concentrations of vitamins $C$ and $E$, plus glutathione, which could protect spermatozoa by inhibiting lipid peroxidation. These authors presented evidence that the sperm storage sites in female birds possess intrinsic antioxidant capabilities owing to elevated activities of glutathione peroxidase and superoxide dismutase. They supported their views by arguing that sperm storage and fertilising abilities can also be improved by supplementation of the diet with extra vitamin $\mathrm{E}$ and selenium. A slightly different perspective was provided by Zaniboni and Bakst, (2004) who demonstrated the presence of aquaporins in avian sperm storage tubules; as these proteins form water channels in cell membranes, these authors proposed that active water exchange between spermatozoa and the storage gland environment might help in the maintenance of sperm viability.

Mammals are also capable of sperm storage in the female reproductive tract, although for shorter periods of time. Sperm storage is particularly well documented in some bat species. Matings take place in the late summer and spermatozoa are stored until fertilisation occurs several months later during the following spring (Racey, 1979; Racey et al, 1987; Racey and Potts, 1970). Morphological observations of sperm disposition within the bat female reproductive tract have revealed that direct contact is frequently established between the uterine epithelium and the sperm head plasma membrane (Wimsatt et al., 1966; Sharifi et al., 2004). These are such intimate contacts that they resemble the interactions between sperm heads and the Sertoli cells of the testis. Nevertheless, in a uterus distended with spermatozoa, the population bound to epithelial cells may represent only a minority of the cells actually present. This casts some doubt on whether the interaction with epithelial cells is essential, or whether the cell-cell interactions are dynamically unstable thus allowing all spermatozoa to reside at the epithelial surface for short periods of time.

Many other mammalian species are able to store spermatozoa, although for periods of days or weeks rather than months. Pioneering experimental insemination studies by Hammond and Asdell (1926) demonstrated that rabbit spermatozoa are capable of surviving in the female reproductive tract for 20-30 hours; for these experiments, insemination was performed without inducing ovulation. A short period of sperm storage in the isthmus region of the oviduct has since been noted in many species (e.g. sheep, Hunter and Nichol, 1983; cow, Lefebvre et al., 1995; pig, Hunter et al., 1984; mare, Boyle et al., 1987; several rodents and marsupials; Taggart and Temple-Smith, 1991; Bedford and Breed, 1994; Esponda and Moreno, 1998).

Although various bat species have adopted their own idiosyncratic means of achieving sperm storage, the other mammals seem to have adopted broadly similar strategies for achieving this effect. Spermatozoa reach the oviductal isthmus having negotiated their way through the uterotubal junction, whereupon they become associated with epithelial cells and a sperm reservoir is formed (for reviews, see Suarez, 1998; Topfer-Petersen et al., 2002). There is some dispute as to the nature of this association; while some authors have reported the establishment of specific sperm-epithelial binding interactions mediated via oligosaccharide groups, others consider that spermatozoa merely become entrapped in oviductal secretions. Prior to ovulation, sperm transport is also physically inhibited; Hunter (2002) commented that viscous glycoproteins form a 
mucus-like plug which almost blocks the distal portion of the isthmus and probably serves several functions, especially the prevention of uterine and ampullary fluids from reaching the sperm reservoir.

The formation of the oviductal sperm reservoir in mammals seems to be important in maintaining sperm viability for a few hours or days. However, unlike the bats, other mammals do not seem to have developed elaborate and semi-permanent sperm-epithelial membrane specialisations, although contact between spermatozoa and epithelial surfaces sometimes, but not always, in crypts, does indeed occur. The nature of the storage mechanism in mammals and other taxa has so far remained elusive; however, the simple physical resemblance between sperm-oviduct interactions in mammals and some of the amphibians and reptiles provides encouragement that eventually general underlying principles might be uncovered. Given that spermatozoa lack the cellular machinery required for de novo protein synthesis, it seems unlikely that wholesale renewal and replacement of sperm components might occur during the storage period. However, in the absence of such replacement the spermatozoa must be vulnerable to damage and degeneration, possibly from oxidative metabolic processes and their products. It therefore seems a reasonable working hypothesis that the important mechanisms may focus on reducing these deleterious effects. Moreover, because the oviductal environment must be largely under epithelial control, it follows that studying the physiology and biochemistry of the epithelial cells must be a sensible starting point for investigating the mechanisms of sperm storage.

Epithelial cell activities may affect closely associated cells in many ways. Not only do they secrete newly synthesised proteins and small peptides into the fluid environment, but they control the ionic composition of the fluid. This is achieved by balancing fluid uptake and export as well as by manipulating ion transport across the apical epithelial cell plasma membrane. Furthermore, while some of the proteins will be soluble in tubal fluid others will become exposed at the epithelial cell surface and will only make direct contact with spermatozoa if they are in close apposition. It is feasible to propose that some of these membrane proteins may participate in cell-cell communication, acting to modulate aspects of sperm metabolism and function. A considerable amount of research has already been undertaken in many of these areas and the interested reader should consult a wealth of previous reviews in this topic (for example: Hunter, 2001; Rodriguez-Martinez et al., 2001; Killian, 2004; Rodriguez-Martinez et al., 2005).

\section{Methodological approaches to sperm-oviduct studies}

Several different approaches to the physiological study of sperm-oviduct interactions have been pursued. Given that the interaction is difficult to study in situ, any in vitro system will possess strengths and weaknesses. It is worth reviewing these briefly as context against which to judge current progress. Some groups have developed methods for obtaining oviduct secretions directly from the in situ oviduct by the use of indwelling catheters (for review, see Killian, 2004) and have both analysed the constituent composition of these fluids and tested their effects on fertilisation and embryonic development. An obvious advantage of this approach is that the nature of the secretions can be correlated accurately with the stage of the reproductive cycle. Achieving this precision of control by in vitro methodology is considerably more problematic. Others have adopted a different approach, namely that of culturing oviductal cells in vitro, and then either observing how spermatozoa interact with the cells (Pacey et al., 1995; Fazeli et al., 1997, 1999; Petrunkina et al., 2001) or recovering conditioned culture medium for testing sperm responses (Kouba et al., 2000). The considerable variety of culture systems employed in 
such studies poses some problems for the interpretation of data. Different systems vary in sophistication; some researchers have used confluent monolayer cultures of isolated oviductal epithelial cells (OEC) while others have been at pains to develop more sophisticated systems in which the epithelial cells retain their natural polarity (Leese et al., 2001). Still others have argued that direct contact between spermatozoa and the oviductal surface is critical in modulating sperm survival and that this is therefore the interaction of greatest interest (Smith and Nothnick, 1997; Elliott et al., 2001a,b; Fazeli et al., 2003).

This disparity of approaches has produced a massive amount of complementary but somewhat confusing evidence about ways in which sperm physiology is controlled by the oviduct. Examination of the literature reveals not only species differences in the way that spermatozoa respond, but also conflicting views on whether capacitation is enhanced or retarded, motility suppressed or activated, and even dispute about whether sperm actually bind to the oviductal surface or not. We have favoured the investigation of mechanisms that involve direct contact, partly because it allows some simplification of the experimental approach. A few comments about interpreting these data may be useful at this point.

The mammalian uterotubal junction acts as a selective barrier to sperm transport; various different types of study have indicated that spermatozoa must be physically intact (i.e. possess intact plasma membrane and acrosome; Esponda and Moreno, 1998), functionally competent at the molecular level (Nakanishi et al., 2004) and appropriately motile. In the latter context it is important to mention the impressive body of literature derived from studies of the $t$-haplotype mouse (Olds-Clarke and Johnson, 1993; Herrmann et al., 1999). In these mice, specific mutations of the $t$-locus cause defective flagellar function that produces transmission ratio distortion in the next generation. This has been attributed to the relative inability of the defective spermatozoa to enter and traverse the uterotubal junction and reach the oviductal isthmus. Selectivity based on other sperm properties, for example the presence or absence of fertilin-ß $\beta$ (Cho et al., 1998), has also been observed. Such rigorous selection means that spermatozoa in the oviductal reservoir will probably also be optimised for storage within a protected site; as a result of their biochemical and structural integrity they probably produce minimal amounts of deleterious free radicals that might cause degeneration of both themselves and their environment.

As spermatozoa in the oviductal reservoir are thus preselected for their stability and integrity, it is likely that continued stabilisation is all that is necessary for further short-term $(<48 \mathrm{~h})$ storage. Contact between spermatozoa and the oviductal epithelial surface seems to be a constant theme across many mammalian species, from mice (Esponda and Moreno, 1998) to marsupials (Bedford and Breed, 1994), so it is reasonable to believe that physical proximity of plasma membranes is involved in the survival mechanism. Although there is much evidence to suggest that oviductal secretions affect capacitation, and even the incidence of polyspermy at fertilisation (Dubuc and Sirard, 1995; Bureau et al., 2000), these effects may be independent of sperm survival in the isthmus and probably act to control sperm function in the ampulla, where fertilisation actually takes place.

\section{Direct cell-cell contact and sperm survival}

For investigators, the study of direct contact between sperm and oviduct poses a number of problems as well as advantages. The main disadvantage is that attempts to elucidate the biochemical interactions almost inevitably involve deconstruction of the in vivo system into what the investigator believes are important component parts; significant uncertainty is associated with the interpretation of data from such studies as we discuss below. From this perspective the 
study of oviductal fluid and its interactions with spermatozoa is less problematic as a research method.

A simple but important source of experimental uncertainty that arises whenever an experiment is set up to investigate sperm-oviductal interactions in vitro is the quality and preparation of spermatozoa to be used. The investigator should probably aim to prepare a subset of high quality spermatozoa for experimental use, thus mimicking the selective activities of the uterotubal junction. This can be achieved to some extent by using selective methods such as Percoll gradient centrifugation or swim-up. Non-selective methods such as washing in a defined medium are probably inadvisable for such experiments on the grounds that they are unphysiological. The use of swim-up separation is also likely to be physiologically inappropriate for many species because it involves exposing the spermatozoa to media typically designed to induce capacitation. Given that oviductal epithelial cells preferentially bind uncapacitated, rather than capacitated, spermatozoa (Fazeli et al., 1999), this sperm preparation method will tend to result in sperm- oviduct membrane binding interactions that may not be relevant. In this context it is also worthwhile considering that where the number of spermatozoa binding to the oviduct has been estimated by in vivo studies, it is surprisingly low in comparison to the numbers inseminated. Mburu et al. (1996) estimated that, in pigs, the uterotubal junction and lower isthmus collectively contained roughly 3000 spermatozoa prior to ovulation; Hunter's estimates of 4000-5000 are almost identical (Hunter, 2002). Compared with the number of spermatozoa in a whole ejaculate, around $300 \mathrm{ml}$ of fluid containing 300 million sperm $/ \mathrm{ml}$ (a total of about 90 $\left.x 10^{9}\right)$, it is easy to see that potentially massive sperm selection pressures are being imposed. As with the mouse studies reported by Esponda and Moreno (1998), Mburu et al, (1996) found by scanning electron microscopy that pig spermatozoa in the oviductal isthmus possessed intact plasma membranes and acrosomes, confirming the view that this is a stringently selected population. In the light of this discussion it is apparent that Percoll (or similar) sperm separation is currently the best experimental approach; however, the development of more sophisticated sperm selection methods holds considerable promise for such experiments (for review, see Suh et al., 2003).

Numerous research groups have studied the interaction of spermatozoa with cultured oviduct cells in vitro, using monolayers, explants and freely suspended cells (Thomas et al., 1995; Dobrinski et al., 1997; Green et al., 2001; Bosch et al., 2001; Gualtieri et al., 2005). This methodology has demonstrated that the cultured cells retain their ability to enhance sperm survival for periods up to several days and that control cells of non-reproductive origin, e.g. kidney, duodenum and lung, cannot match this ability. These experiments have also shown that the initial sperm binding event is mediated via specific oligosaccharide residues; in pigs, the appropriate carbohydrate recognition molecules are synthesised in the seminal vesicles and prostate and transferred to the sperm surface via seminal plasma (Topfer-Petersen et al., 2002). This initial binding is reminiscent of the sperm-oocyte interaction, where primary and secondary binding events take place. It is feasible that the sperm-oviduct interactions also involve secondary binding in which other receptors and ligands are able to express their functional importance.

Smith and Nothnick (1997) were among the first to demonstrate that direct contact with apical plasma membrane (APM) vesicles prepared from the rabbit oviduct are able to enhance sperm viability in vitro. Interestingly, they were also able to show that the effect was a specific property of the oviduct, other types of epithelia being unable to serve as substitutes. Moreover, their experiments demonstrated that the epithelial plasma membrane vesicles alone were sufficient for this effect to occur. De novo protein synthesis by epithelial cells can therefore be ruled out as an essential step in the mediation of sperm survival, although other studies have 
shown that sperm binding nevertheless induces new gene expression (Thomas et al., 1995; Fazeli et al., 2004).

Careful consideration of Smith and Nothnick's (1997) demonstration that membrane vesicles prepared from the oviduct had the ability to enhance sperm survival suggests that regulatory receptor-ligand interactions are probably being recruited to control sperm function. Spermatozoa are well known to possess myriads of membrane receptors, most whose functions are at best still unclear; this led Meizel (2004) to call them "neurones with a tail". Capacitation and the acrosome reaction have been identified as being promoted by receptor-ligand interactions, so it is conceivable that inhibition of capacitation and of the acrosome reaction are controlled in the same way, and that appropriate ligands are exposed within the isolated membrane fractions. This hypothesis was investigated further by Fazeli et al., (2003) who, in an effort to focus on the membrane proteins in more detail, prepared soluble fractions of porcine APM (SAPM) and showed that these also possessed the ability to prolong the survival of boar spermatozoa in vitro. In these studies the SAPM was derived from the membrane vesicles by extraction with a high concentration $(1 \mathrm{M})$ of sodium chloride. As this treatment corresponds to the textbook definition of peripheral membrane proteins, being tightly bound to a membrane but not integrated into its structure, these findings support the hypothesis that direct contact with spermatozoa is particularly important in maintaining sperm viability and integrity in vivo. Based on these results we are currently examining sperm-membrane protein interactions in more detail to identify the most significant receptors and ligands.

The interactions of bovine spermatozoa with oviductal cell apical plasma membrane components have also been studied in considerable detail by Boilard et al., (2002). These authors, in addition to confirming that the interaction prolongs sperm viability, showed that the isolated membrane fraction was capable of controlling intracellular calcium concentrations and preventing individual spermatozoa from reaching lethal calcium levels. More recently these authors have also shown, by the use of radiolabelled membrane fractions, that the spermatozoa engage in tight binding with several membrane proteins; one of these was identified as a heat shock protein (HSP60) and another as glucose-regulated protein 78 (GRP78). A possible role of heat shock proteins in the enhancement of sperm viability is to be taken seriously as these proteins engage in protein repair processes when cells are exposed to stresses. They have been identified in other studies of the oviduct, and are therefore credible participants in the sperm-oviduct interaction. Nevertheless, the technical considerations outlined above should be taken into account when interpreting these results. The spermatozoa used in the experiments were not selected for integrity or quality, and had in fact been cryopreserved, thus changing their surface characteristics. Furthermore, there is a possibility that the radiolabelled proteins could have originated on the cytoplasmic face of the apical plasma membrane, thus not normally engaging in sperm-oviduct interactions.

\section{Oviductal regulation of sperm motility and capacitation}

Several recent studies have provided evidence that sperm-oviduct interactions resuit in signal transduction processes that control capacitation. A study by Gualtieri et al., (2005) illustrated two significant aspects of this interaction, namely that when bull spermatozoa are bound to oviductal cell monolayers in vitro they exhibit both low intracellular calcium concentrations and low levels of tyrosine phosphorylation of proteins. Elevation of intracellular calcium concentration and tyrosine phosphorylation are two widely recognised indications of capacitation, and so it is also significant that in their study Gualtieri et al., (2005) also showed that sperm release from the oviductal surface involved both processes. Comparable data, derived from 
studies of porcine and equine oviductal cells, have been also been reported previously (Dobrinski et al., 1997; Topfer-Petersen et al., 2002).

In similar vein, significant downregulation of another crucial aspect of sperm function, namely motility, has also been reported by a number of authors (e.g.; Overstreet and Cooper, 1975; Grippo et al., 1995). In our laboratory we are currently using soluble APM preparations to see whether this involves specific ligand-receptor interactions. Boar sperm motility is unusually sensitive to environmental effects, especially the presence of bicarbonate, and much has been written about it in relation to fluidisation of the plasma membrane, the rapid elevation of intracellular cAMP concentration (Harrison et al., 1993a,b; Harrison et al., 1996), protein kinase activation (Harrison, 2004) and the way in which boar sperm motility increases rapidly in vitro ( $<2 \mathrm{~min}$ ) when bicarbonate is added to suspensions (Holt and Harrison, 2002). At face value this last observation, which is indeed very striking (Fig. 1), appears at variance with reports of motility suppression in the oviduct, where bicarbonate concentration is reportedly rather high (approximately 35mM; Rodriguez-Martinez et al., 2005).

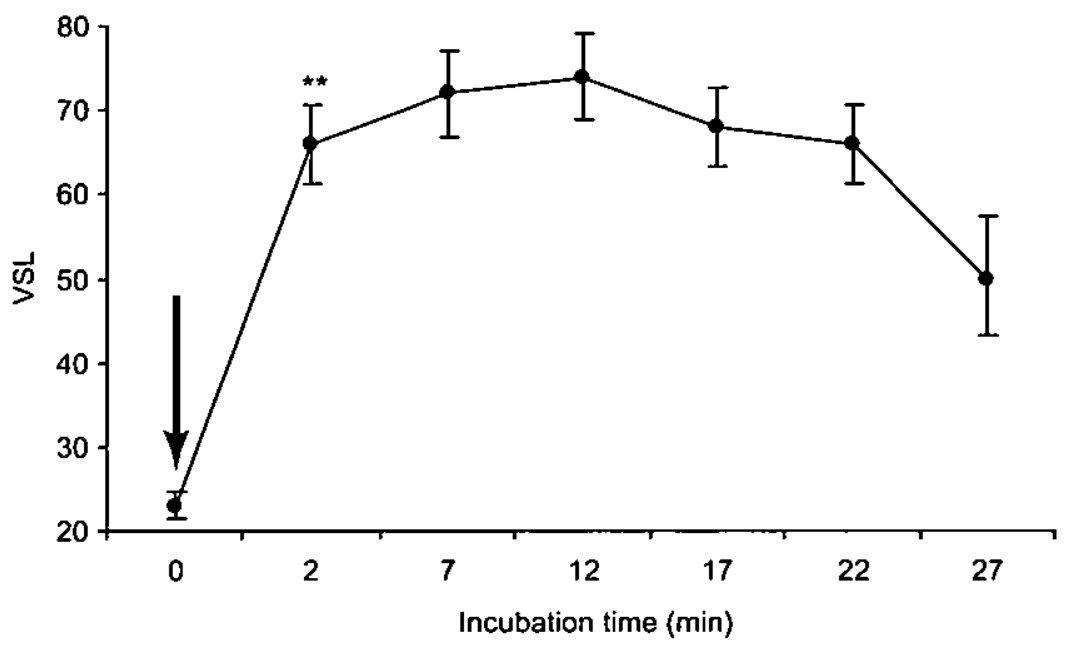

Fig. 1. Illustration of bicarbonate-induced increase in linear velocity of boar spermatozoa. Percoll washed spermatozoa from a single ejaculate were pre-incubated in Tyrode's medium (lacking bicarbonate) for $10 \mathrm{~min}$ before the addition of $15 \mathrm{mM}$ bicarbonate/ $\mathrm{CO}_{2}$. Subsamples were taken for videorecordings of motility shortly before ("arrow") and at intervals after bicarbonate addition. Controls supplemented with appropriate amounts of $\mathrm{NaCl}$ were sampled 27 minutes after the addition of $15 \mathrm{mM}$ bicarbonate/CO (data not shown). Motility parameter values were obtained by analysis of individual sperm tracks using the Hobson Sperm Tracker (For detailed method, see Holt and Harrison, 2002). The graph shows the mean straight-line velocity (VSL) for all the motile sperm analysed at each time point ( 200 cells per time point); the bars show the $95 \%$ confidence intervals of the means. Significantly increased VSL is apparent 2 minutes after bicarbonate addition but it then reaches a maximum at 12 minutes and velocity declines thereafter $\left(^{* *}\right.$ : different from "zero-time" value, $P<0.005$ ).

In keeping with the arguments presented above, we hypothesise that oviductal components can interact with uncapacitated spermatozoa to suppress their motility, despite the presence of bicarbonate. Our preliminary data (Satake et al., 2005) supports this hypothesis, showing that the soluble APM preparation described above is indeed capable of reducing the motility activa- 
tion response (Fig. 2). Sperm motility was measured quantitatively using computerised semen assessment technology (CASA), and it is clear that when Percoll-washed boar spermatozoa are stimulated with $15 \mathrm{mM}$ bicarbonate $/ \mathrm{CO}_{2}$, the inclusion of $100 \mu \mathrm{g} / \mathrm{ml}$ sAPM can reduce the degree of stimulation. The same abstract also describes an accompanying experiment establishing that the effect is not achieved simply by blocking the uptake of bicarbonate by spermatozoa, because intracellular $\mathrm{pH}$ became elevated in tandem with incubation time. These results are consistent with the modulation of signal transduction pathway(s), but at present we cannot be certain of the pathways involved.

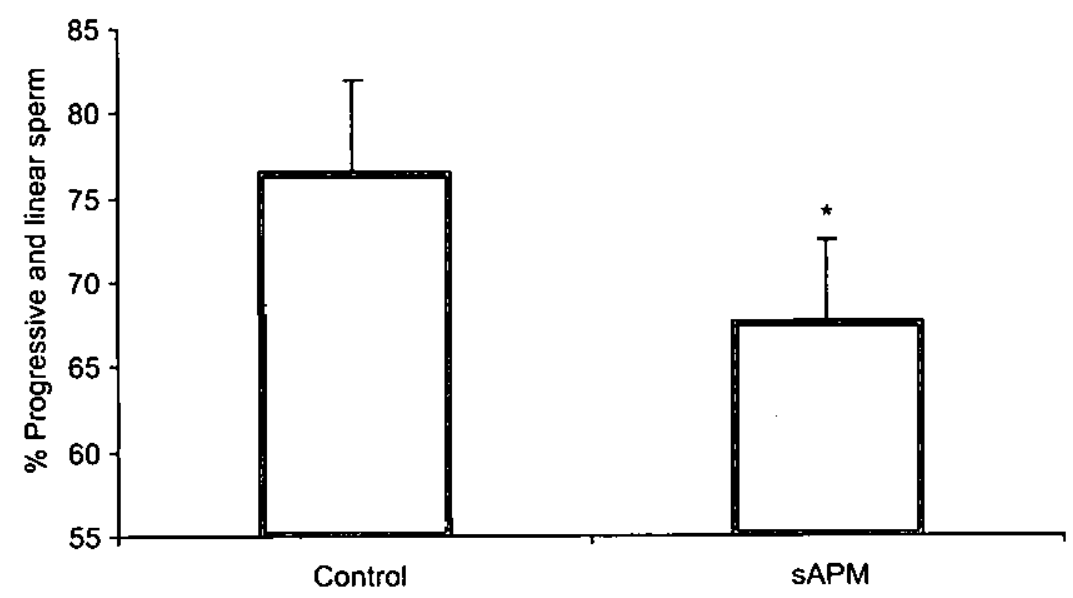

Fig. 2. Using the same methodology as described for Figure 1, the stimulation effect of bicarbonate was tested on 9 separate samples of washed boar semen and compared with equivalent effects in paired samples treated with $100 \mu \mathrm{g} / \mathrm{ml}$ SAPM (solubilised apical plasma membrane fraction; Fazeli et al., 2000). Analysis of the sperm motility response was undertaken using cluster analysis of four kinematic parameters to examine sperm subpopulation responses. Inclusion of SAPM in the experimental medium reduced the bicarbonate-induced sperm activation response, characterised in this graph as the proportion of linear and progressive spermatozoa observed after activation (ANOVA; $F_{1 / 148}$; $P=0.024)$.

Some recent evidence about the suppressive control of boar sperm motility is, however, pertinent in this respect. Recently Aparicio et al. (2005) demonstrated that boar sperm motility can be downregulated via the phosphatidylinositol 3-kinase (PI-3 kinase) signal transduction pathway, which appears to counterbalance activation via the bicarbonate-sensitive, protein kinase A-stimulated pathway. These two pathways, if differentially active in the oviduct, are certainly capable of positively and negatively controlling boar sperm motility.

\section{Prospects for the exploitation of oviductal function for biotechnology}

In the discussion above, we have deliberately concentrated on the natural mechanisms that enhance sperm survival in the oviductal reservoir by turning off a number of their functions. An extensive literature shows that the suppression of sperm function is reversed around the time of ovulation, with the expression of activated and hyperactivated motility, changes in membrane lipid architecture and fluidity, upregulation of protein phosphorylation and even the possibility 
that spermatozoa become sensitive to chemotactic signals emanating from oocytes (Eisenbach, 1999). Switching from one state to another is a physiological response, possibly dominated by the endocrine background, but also modulated by the spermatozoa themselves which stimulate de novo protein synthesis within the oviduct (Ellington et al., 1993; Thomas et al., 1995; Fazeli et al., 2004).

The oviductal system in mammals appears to offer a variety of opportunities that may be open to exploitation for improving sperm survival and artificial insemination. Most seem to be based around signal transduction activities that are an evident feature of sperm-oviduct interactions. Emerging evidence that sperm metabolism, capacitation and motility can be turned off and on as required, suggests that, in principle, the prospects for long-term sperm survival seem very promising. Combining this fundamental level of control with the use of anti-oxidants, some of which are already known to operate within the oviduct (e.g. catalase; Lapointe et al., 1998), could very well see the extension of in vitro sperm lifespan many days beyond that currently achievable. Although this is still rather speculative, it nevertheless represents informed speculation and is more soundly based than the mainly empirical approach to sperm diluent development that has traditionally been employed.

Although oviductal proteins appear to be the important modulators of sperm function, biosecurity and practicality will dictate that for commercial purposes it will not be possible to isolate and use oviductal proteins from slaughtered animals as sources of materials. This has important implications for research, because we must not only determine the nature of the biological processes that control sperm function, we will have to identify the relevant receptors and ligands so that modulators can be synthesised and produced on a large scale. Although we are still at the first stage, advances in proteomics, crystallography and molecular modelling should enable us to progress to the second stage once we have a series of confirmed targets. This is very much the domain of the new "Biotech" industries, which depend on intellectual property rights for survival; further significant progress may therefore lie in the hands of commercial enterprises rather than academic scientists.

Besides seeking ways to extend the lifespan of all spermatozoa, logically we should also imitate the oviduct and try to select the best subpopulation for long-term storage. As a minimum, the oviduct is selecting spermatozoa on the basis of cellular integrity and motility; exclusion of damaged cells may help to minimise the production of free radicals and the release of lytic enzymes from damaged acrosomes. At present, busy centres process semen on an industrial scale and so the introduction of sperm selection procedures may seem highly inconvenient. However, with the gradual introduction of deep intrauterine insemination procedures that require smaller insemination volumes and fewer sperm, selecting high quality sperm may be less of a technical imposition.

\section{Conclusions}

In this review we have demonstrated that aspects of oviductal function concerned with the maintenance of sperm viability are remarkably conserved across a range of vertebrates, and uncouple the synchrony between insemination timing and ovulation. Given the extremely long storage periods accomplished by some species, especially reptiles, it is clear that important mechanisms for the prolongation of sperm viability operate successfully and outperform any semen diluent technologies developed to date. Although insufficient data is available about cell signalling pathways and capacitation in species such as reptiles, analysis of the literature implies that this level of performance is likely to be achieved through cell signalling pathways that tend to prevent both fertilisation and premature cell death. Sperm motility sup- 
pression is a common feature of natural sperm storage mechanisms; if there is no immediate requirement for sperm motility then it makes energetic sense to suppress it, together with the associated requirement for substrates. Intuitively this seems to be a sensible policy for the design of long-life semen diluents. Indeed, anyone who examines boar spermatozoa in BTS medium will observe that their motility is somewhat poor. Unfortunately, this principle is poorly understood by some Al practitioners, who mistakenly equate the poor motility with poor quality.

We have not addressed the likelihood that sperm repair mechanisms might also be in operation, but this should not be overlooked. Recent evidence that boar sperm quality may be correlated with the presence of chaperonins and heat shock proteins (Huang et al., 1999, 2000) coupled with the identification of HSP60, one of the oviduct membrane components that bind avidly to spermatozoa (Boilard et al., 2004), provides food for thought. These proteins exist for their protective abilities and it would be unsurprising if they were significant players in the maintenance of sperm viability.

\section{Acknowledgements}

We thank Defra, BBSRC, Genus Breeding Ltd and Sygen International Plc for their financial support.

\section{References}

Almeida-Santos SM, Laporta-ferreira IL, Antoniazzi MM and Jared $C(2004)$ Sperm storage in males of the snake Crotalus durissus terrificus (Crotalinae: Viperidae) in southeastern Brazil Comparative Biochemistry and Physiology A Molecular and Integrative Physiology 139 169-174

Aparicio IM, Gil MC, Garcia-Herreros M, Pena Fl and Garcia-Marin L) (2005) Inhibition of phosphatidylinositol 3-kinase modifies boar sperm motion parameters Reproduction 129 283-289

Bakst MR (1993) Oviducal sperm storage in poultry: a review Reproduction Fertility and Development 5 595-599

Bakst MR and Vinyard BT (2002) Oviducal sperm storage in turkeys: spatial distribution of sperm within the uterovaginal junction sperm-storage tubules fournal of Experimental Zoology 292 206-209

Bedford JM and Breed WG (1994) Regulated storage and subsequent transformation of spermatozoa in the Fallopian tubes of an Australian marsupial, Sminthopsis crassicaudata Biology of Reproduction 50 845-854

Birkhead TR (1998) Sperm competition in birds Reviews in Reproduction 3 123-129

Boilard M, Bailey J, Collin S, Dufour M and Sirard M-A (2002) Effect of bovine oviduct epithelial cell apical plasma membranes on sperm function assessed by a novel flow cytometric approach Biology of Reproduction 67 1125-1132

Boilard M, Reyes-Moreno C, Lachance C, Massicotte L, Bailey IL, Sirard MA and Leclerc P (2004) Localization of the chaperone proteins GRP78 and HSP60 on the luminal surface of bovine oviduct epithelial cells and their association with spermatozoa Biology of Reproduction 71 1879-1889

Bosch P, de Avila JM, Ellington JE and Wright RW, Jr. (2001) Heparin and $\mathrm{Ca} 2+$-free medium can enhance release of bull sperm attached to oviductal epithelial cell monolayers Theriogenology 56 247-260

Boyle MS, Cran DG, Allen WR and Hunter RH (1987) Distribution of spermatozoa in the mare's oviduct Journal of Reproduction and Fertility Supplement 35 79-86

Breque C, Surai P and Brillard IP (2003) Roles of antioxidants on prolonged storage of avian spermatozoa in vivo and in vitro Molecular Reproduction and Development 66 314-323

Bureau M, Bailey JL and Sirard MA (2000) Influence of oviductal cells and conditioned medium on porcine gametes Zygote 8 139-144

Cho C, Bunch DO, Faure JE, Goulding EH, Eddy EM, Primakoff $P$ and Myles DG (1998) Fertilization defects in sperm from mice lacking fertilinß Science 281 1857-1859

Dobrinski I, Smith TT, Suarez SS and Ball BA (1997) Membrane contact with oviductal epithelium modulates the intracellular calcium concentration of equine spermatozoa in vitro Biology of Reproduction 56 861-869

Dubuc A and Sirard MA (1995) Effect of coculturing spermatozoa with oviductal cells on the incidence of polyspermy in pig in vitro fertilization Molecular Reproduction and Development $41360-367$

Eisenbach $M$ (1999) Mammalian sperm chemotaxis and 
its association with capacitation Developmental Genetics 25 87-94

Ellington JE, Ignotz GG, Ball BA, Meyerswallen VN and Currie WB (1993) De novo protein-synthesis by bovine uterine tube (oviduct) epithelial-cells changes during coculture with bull spermatozoa Biology of Reproduction 48 851-856

Elliott RMA, Duncan A, Watson PF, Holt WV and Fazeli AR (2001a) Enhanced boar sperm viability with porcine oviductal apical plasma membranes (APM) Reproduction Abstract series No. 2733

Elliott RMA, Duncan A, Watson PF, Holt WV and Fazeli AR (2001b) Peripheral bound membrane proteins are involved in the maintenance of boar sperm viability by oviductal apical plasma membrane preparations in vitro Molecular Biology of the Cell $12117 \mathrm{a}$

Esponda $P$ and Moreno $M$ (1998) Acrosomal status of mouse spermatozoa in the oviductal isthmus lournal of Experimental Zoology 282 360-366

Fazeli A, Affara NA, Hubank M and Holt WV (2004) Sperm-induced modification of the oviductal gene expression profile after natural insemination in mice Biology of Reproduction 71 60-65

Fazeli A, Duncan AE, Watson PF and Holt WV (1999) Sperm-oviduct interaction: induction of capacitation and preferential binding of uncapacitated spermatozoa to oviductal epithelial cells in porcine species Biology of Reproduction $60879-886$

Fazeli A, Elliott RM, Duncan AE, Moore A, Watson PF and Holt WV (2003) in vitro maintenance of boar sperm viability by a soluble fraction obtained from oviductal apical plasma membrane preparations Reproduction 125 509-517.

Fazeli A, Watson PF and Holt WV (1997) Assessment of a porcine sperm-oviduct culture system using frozen oviductal cells Journal of Reproduction and Fertility Abstract Series 2049

Galbraith DA (1993) Multiple paternity and sperm storage in turtles Herpetology Journal $3117-123$

Gist DH and Congdon JD (1998) Oviductal sperm storage as a reproductive tactic of turtles journal of Experimental Zoology 282 526-534

Gist DH and Jones JM (1987) Storage of sperm in the reptilian oviduct Scanning Microscopy 1 1839-1849

Green CE, Bredl J, Holt WV, Watson PF and Fazeli A (2001) Carbohydrate mediation of boar sperm binding to oviductal epithelial cells in vitro Reproduction 122 305-315.

Grippo AA, Way AL and Killian GJ (1995) Effect of bovine ampullary and isthmic oviductal fluid on motility, acrosome reaction and fertility of bull spermatozoa Journal of Reproduction and Fertility 105 57-64

Gualtieri R, Boni R, Tosti E, Zagami $M$ and Talevi $R$ (2005) Intracellular calcium and protein tyrosine phosphorylation during the release of bovine sperm adhering to the Fallopian tube epithelium in vitro Reproduction 129 51-60

Hammond J and Asdell SA (1926) The vitality of the spermatozoa in the male and female reproductive tracts The British Journal of Experimental Biology 4 155-185
Harrison RA (2004) Rapid PKA-catalysed phosphorylation of boar sperm proteins induced by the capacitating agent bicarbonate Molecular Reproduction and Development 67 337-352

Harrison RAP, Ashworth PJC and Miller NGA (1993a) Rapid effects of bicarbonate/ $\mathrm{CO}_{2}$ on boar spermatozoa detected by merocyanine, a probe of lipid packing Journal of Reproduction and Fertility Abstract Series 12 Abstract 18

Harrison RAP, Ashworth PJC and Miller NCA (1996) Bicarbonate/CO, an effector of capacitation, induces a rapid and reversible change in the lipid architecture of boar sperm plasma membrane Molecular Reproduction and Development 45 378-391

Harrison RAP, Mairet $B$ and Miller NGA (1993b) Flow cytometric studies of bicarbonate-mediated $\mathrm{Ca}^{2+}$ influx in boar sperm populations Molecular Reproduction and Development 35 197-208

Herrmann BG, Koschorz B, Wertz K, Mclaughlin KJ and Kispert A (1999) A prolein kinase encoded by the t complex responder gene causes non- Mendelian inheritance Nature 402 141-146.

Holt WV and Harrison RA (2002) Bicarbonate stimulation of boar sperm motility via a protein kinase Adependent pathway: between-cell and betweenejaculate differences are not due to deficiencies in protein kinase A activation Journal of Andrology 23 557-565

Huang SY, Kuo YH, Lee WC, Tsou HL, Lee YP, Chang HL, Wu J/ and Yang PC (1999) Substantial decrease of heat-shock protein 90 precedes the decline of sperm motility during cooling of boar spermatozoa Theriogenology 51 1007-1016

Huang SY, Kuo YH, Lee YP, Tsou HL, Lin EC, Ju CC and Lee WC (2000) Association of heat shock protein 70 with semen quality in boars Animal Reproduction Science 63 231-240

Hunter RH (2001) Histophysiology of the Fallopian tubes in relation to sperm binding, release, and completion of capacitation Italian Journal of Anatomy and Embryology 106 279-289

Hunter RH (2002) Vital aspects of Fallopian tube physiology in pigs Reproduction in Domestic Animals 37 186-190

Hunter RHF, Flechon B and Flechon JE 1984 Pre-ovulatory arrest and peri-ovulatory redistribution of competent spermatozoa in the isthmus of the pig oviduct Journal of Reproduction and Fertility 72 203-211

Hunter RH and Nichol R (1983) Transport of spermatozoa in the sheep oviduct: preovulatory sequestering of cells in the caudal isthmus fournal of Experimental Zoology 228 121-128

Killian GJ (2004) Evidence for the role of oviduct secretions in sperm function, fertilization and embryo development Animal Reproduction Science 82-83 141-153

Kouba Al, Abeydeera LR, Alvarez IM, Day BN and Buhi WC (2000) Effects of the porcine oviduct-specific glycoprotein on fertilization, polyspermy, and embryonic development in vitro Biology of Reproduction $63242-250$ 
Lapointe S, Sullivan R and Sirard MA (1998) Binding of a bovine oviductal fluid catalase to mammalian spermatozoa Biology of Reproduction 58 747-753

Leese HJ, Tay JI, Reischl J and Downing SJ (2001) Formation of Fallopian tubal fluid: role of a neglected epithelium Reproduction 121 339-346

Lefebvre R, Chenoweth PJ, Drost $M$, LeClear CT, MacCubbin M, Dutton JT and Suarez SS (1995) Characterization of the oviductal sperm reservoir in cattle Biology of Reproduction 53 1066-1074

Mburu JN, Einarsson S, Lundeheim $\mathbf{N}$ and RodriguezMartinez H (1996) Distribution, number and membrane integrity of spermatozoa in the pig oviduct in relation to spontaneous ovulation Animal Reproduction Science 45 109-121

Meizel S (2004) The sperm, a neuron with a tail: 'neuronal' receptors in mammalian sperm Biological Reviews of the Cambridge Philosophical Society 79 713732

Nakanishi T, Isotani A, Yamaguchi R, Ikawa M, Baba T, Suarez SS and Okabe $M$ (2004) Selective passage through the uterotubal junction of sperm from a mixed population produced by chimeras of calmeginknockout and wild-type male mice Biology of Reproduction 71 959-965

Olds-Clarke P and Johnson LR (1993) T-haplotypes in the mouse compromise sperm flagellar function Developmental Biology 155 14-25

Overstreet IW and Cooper GW (1975) Reduced sperm motility in the isthmus of the rabbit oviduct Nature 258 718-719

Pacey AA, Hill CJ, Scudamore IW, Warren MA, Barratt CLR and Cooke ID (1995) The interaction in vitro of human spermatozoa with epithelial cells from the human uterine (Fallopian) tube Human Reproduction 10 360-366

Pearse DE, Janzen FJ and Avise JC (2001) Genetic markers substantiate long-term storage and utilization of sperm by female painted turtles Heredity 86378 . 384

Petrunkina AM, Gehthaar R, Drommer W, Waberski D and Topfer-Petersen E (2001) Selective sperm binding to pig oviductal epithelium in vitro Reproduction $121889-896$

Pratt HL, Jr. and Tanaka S (1994) Sperm storage in male elasmobranchs: a description and survey journal of Morphology 219 297-308

Racey PA (1979) The prolonged storage and survival of spermatozoa in Chiroptera lournal of Reproduction and Fertility 56 391-402

Racey PA and Potts DM (1970) Relationship between stored spermatozoa and the uterine epithelium in the pipistrelle bat (Pipistrellus pipistrellus) Journal of Reproduction and Fertility 22 57-63

Racey PA, Uchida TA, Mori T, Avery MI and Fenton MB (1987) Sperm-epithelium relationships in relation to the time of insemination in little brown bats (Myotis lucifugus) Journal of Reproduction and Fertility $\mathbf{8 0}$ 445-454

Rodriguez-Martinez $H$, Saravia F, Wallgren $M$, Tienthai P, Johannisson A, Vazquez JM, Martinez E, Roca J, Sanz L and Calvete JJ (2005) Boar spermatozoa in the oviduct Theriogenology $63514-535$

Rodriguez-Martinez H, Tienthai P, Suzuki K, Funahashi $H$, Ekwall $H$ and Johannisson $A$ (2001) Involvement of oviduct in sperm capacitation and oocyte development in pigs Reproduction Supplement 58 129145

Satake N, Watson PF and Holt WV (2005) How does the oviduct control sperm motility? 7th international Conference on Pig Reproduction, Kerkrade, The Netherlands. Abstract 124.

Sever DM and Brizzi R (1998) Comparative biology of sperm storage in female salamanders fournal of Experimental Zoology 282 460-47

Sharifi M, Ghorbani R, Fazeli A and Holt WV (2004) Evidence of sperm storage in Pipistrellus kuhlii (Chiroptera:Vespertilioniodae) in western Iran Folia Zoologica 52 1-6

Smith TT and Nothnick WB (1997) Role of direct contact between spermatozoa and oviductal epithelial cells in maintaining rabbit sperm viability Biology of Reproduction 56 83-89.

Suarez SS (1998) The oviductal sperm reservoir in mammals; mechanisms of formation Biology of Reproduction 58 1105-1107

Suh RS, Phadke N, Ohl DA, Takayama S and Smith GD (2003) Rethinking gamete/embryo isolation and culture with microfluidics Human Reproduction Update 9 451-461

Taggart DA and Temple-Smith PD (1991) Transport and storage of spermatozoa in the female reproductive tract of the brown marsupial mouse, Antechinus stuartii (Dasyuridae) Journal of Reproduction and Fertility 93 97-110

Thomas PG, Ignotz GG, Ball BA, Brinsko SP and Currie WB (1995) Effect of coculture with stallion spermatozoa on de novo protein synthesis and secretion by equine oviduct epithelial cells American Journal of Veterinary Research 56 1657-1662

Topfer-Petersen E, Wagner A, Friedrich J, Petrunkina A, Ekhlasi-Hundrieser $M$, Waberski $D$ and Drommer $W$ (2002) Function of the mammalian oviductal sperm reservoir Journal of Experimental Zoology 292210 215

Wimsatt WA, Krutzsch PH and Napolita L (1966) Studies on sperm survival mechanisms in female reproductive tract of hibernating bats. I. Cytology and ultra-structure of intra-uterine spermatozoa in Myotis L.ucifugus American Journal of Anatomy 119 25-60

Zaniboni L and Bakst MR (2004) Localization of aquaporins in the sperm storage tubules in the turkey oviduct Poultry Science 83 1209-1212 